\title{
Acute pancreatitis caused by postcholecystectomic pseudoaneurysmatic hemobilia
}

Tamzeed Hossain ${ }^{1 *}$, Nazmun Nahar Munny², Chowdhury Rifat Niger ${ }^{3}$, Hasan Tasmim¹, Rawshan Arra $\mathrm{Khanam}^{4}$, Jan Mohammad ${ }^{5}$, Mohammed Mahbub Alam ${ }^{6}$

\begin{abstract}
:
A 50 year old bangladeshi female, came to our emergency with hematemasis, jaundice and abdominal pain who had a history of laparoscopic cholecystectomy 1 month ago. Patient was diagnosed as acute pancreatitis and obstructive jaundice caused by postcholecystectomic hemobilia. She also had a vascular abnormaly (Her left lobe of liver is supplied by hepatic artery and right love of liver is supplied by accessory hepatic artery which is a branch of superior mesenteric artery, and a sacular aneurysm developed in accessory hepatic artery near the gall bladder fossa (near postcholecystectomy clipping). We are reporting another case of acute pancreatitis after laparoscopic cholecystectomy caused by hemobilia secondary to pseudoaneurysm in accessory hepatic artery originating from superior mesenteric artery. This is probably second such reported case.
\end{abstract}

Keywords: Hemobilia, Pseudoaneurysm, acute pancreatitis, LC (Laparoscopic cholecystectomy), NJ tube (najojejunal tube), ERCP (Endoscopic retrograde cholangiopancreatography), OGD (oesophagogastrodudenoscopy), Quinke's triad.

\section{Background:}

Haemobilia is bleeding into billiary tree. It is characterized by the Quinke's triad of abdominal pain, jaundice, and an upper gastrointestinal (UGI) bleed, which can be present in approximately $25 \%$ of cases. Aneurysmal disease of visceral arteries is uncommon, and it usually involves branches of the hepatic and gastro-duodenal arteries. ${ }^{1}$ Hemobilia is an uncommon cause of upper gastrointestinal bleeding and can occur following liver biopsy, transjugular intrahepatic portosystemic shunt (TIPS) placement, trauma, hepatocellular carcinoma, cholangiocarcinoma, gallstones, pancreatitis, polyarteritis nodosa, biliary ascariasis, and hepatic artery aneurysm. $^{2}$

\section{Case report:}

A 50 Years Bangladeshi female presented in emergency with

1. Senior House officer, Department of Gastroenterology, United hospital limited, Gulshan-02, Dhaka, Bangladesh

2. Post graduate student, M-Phil-Thesis part-(Microbiology), Dhaka medical college Hospital.

3. Specialist, Dept. of Gastroenterology, United hospital limited, Gulshan-02, Dhaka, Bangladesh.

4. Consultant Pulmonologist, Ingenious Health Care Ltd, Dhaka Bangladesh

5. Consultant,Dept.of Radiology \& imaging, United hospital limited, Gulshan-02, Dhaka, Bangladesh

6. Consultant, Dept. of Gastroenterology, United hospital limited, Gulshan-02, Dhaka, Bangladesh

*Corresponding Author:

Dr. Tamzeed Hossain

Senior House officer, Department of Gastroenterology, United hospital limited, Gulshan-02, Dhaka, Bangladesh

Email: Tamzeed.riyad@gmail.com

Contact: +8801937139271 the complaints of severe upper abdominal pain for two days $\&$ several episodes of haematemasis \& melaena for one day. History reveled that she had acute pancreatitis due to cholelithiasis two months ago. She underwent elective laparoscopic cholecystectomy one month back in outside hospital. Her operative note showed that cholecystectomy was completed without any difficulty \& her postcholecystectomy status was uneventful. On physical examination, patient was toxic, anaemic, icteric, tachycardic (112/min), blood pressure-110/60mm of $\mathrm{Hg} \&$ body temperature $101^{\circ} \mathrm{F}$. She had tenderness \& guarding in the epigastric ,right hypochondrium and umbilical region. Liver dullness was not obliterated, and there were no signs of generalized peritonitis. Patient got two units of whole blood in outside hospital before admitting here and oesophagogastrodudenoscopy (OGD) was tried to find out the bleeding source but that attempt failed to identify. Our laboratory result showed $\mathrm{Hb} \%-9.0 \mathrm{mg} / \mathrm{dl}$, Leucocytes-10600cells $/ \mathrm{mm}^{3}$, total bilirubin- $6.84 \mathrm{mg} / \mathrm{dl}$, ALT-194 IU/L, AST-180 IU/L, ALP-250 IU/L, S.Amylase-812I U/L, S.Lipase-2287 IU/L, her coagulation profile was normal. Acute pancreatitis was evident. Abdominal utrasonography did not demonstrate any pathological findings except confirming that patient is cholecystectomised. During diagnostic evaluation period, intravenous fluid resuscitation was made $\&$ hemoglobin level of the patient was brought to $11 \mathrm{mg} / \mathrm{dl}$, by 2 units of whole blood transfusion. Upper GI endoscope was done 12 hours after admission to find out the bleeding source \& for giving NJ tube for feeding management.A side view endoscopy identified blood leakage through duodenal papilla (Fig-1). We did not perform sphincterotomy to evacuate the clot or a cholangiogram from fear of precipitating massive bleeding. MRCP was performed immediately and MRCP showed CBD 
mildly dilated and lumen is filled up by altered signal intensity area - suspected collection, intrahepatic billiary channels are mildly dilated, the pancrease is swollen but pancreatic ducts are not dilated, the gall bladder is not visualized (H/O-cholecystectomy). At that day abdominal computerized tomography and angiography done which detects arterial supply of the left lobe of the liver by the hepatic artery and the right lobe of liver by the accessory hepatic artery- arising from the superior mesenteric artery. Sacullar aneurysm measuring about $2.1 \mathrm{~cm} \times 1.5 \mathrm{~cm}$ is noted in the region of gall bladder fossa arising from that accessory hepatic artery supplying the right lobe. Intrahepatic billiary channels were prominent, extrahepatic bile duct was mildly dilated and lumen was hyperdense. Pancrease mildly swollen, pancreatic ducts were mildly dilated (Figure-2 \& 3).

ERCP \& angiographic embolization was planned. However, he was taken to another hospital for financial constraint. He died there before having any intervention.

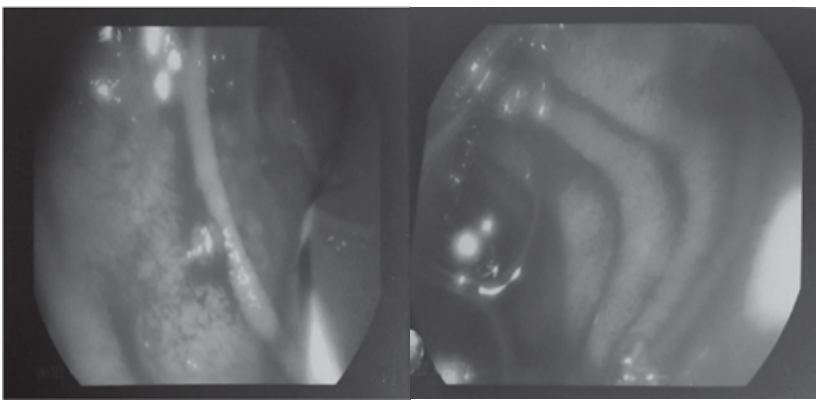

Figure1: Endoscopic view showing the blood coming out through duodenal papilla

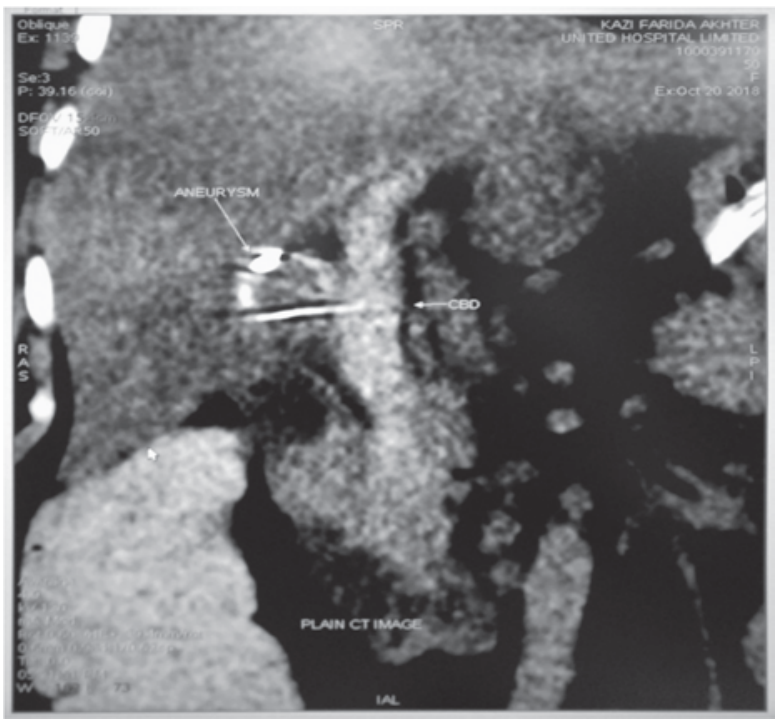

Figure 2: Coronal section of computed tomography showing psuedoaneurysm from accessory hepatic artery and dilated CBD

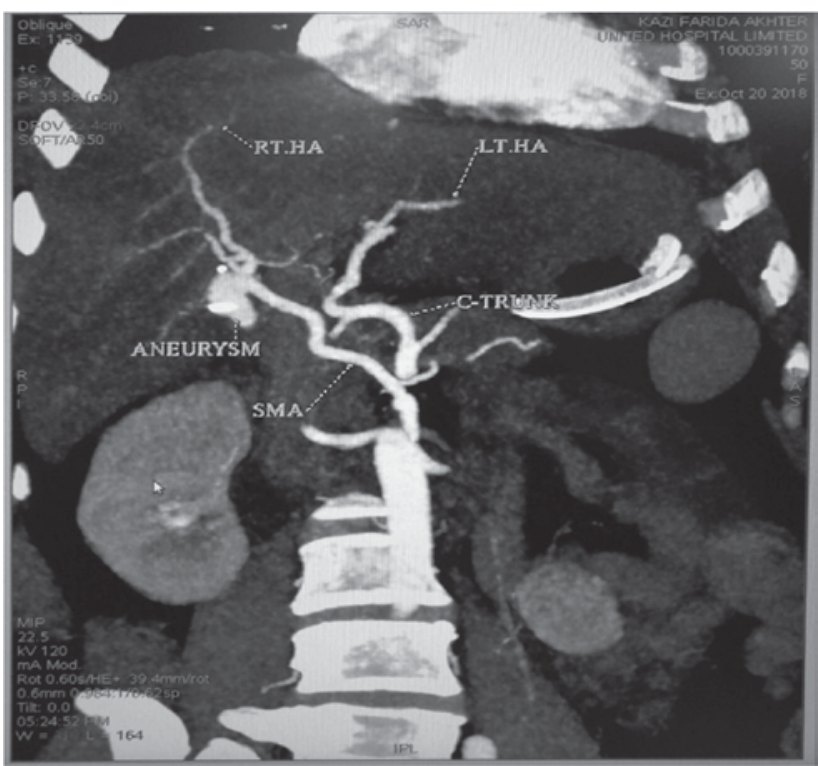

Figure 3: CT angiography showing psuedoaneurysm from accessory hepatic artery

\section{Discussion}

Hemobilia to describe bleeding into the biliary system after a sub capsular liver injury but this term now covers all reasons which causes bleeding in to the biliary tree. The term was first used by Sandblom. ${ }^{3}$ It is a rare cause of upper gastrointestinal hemorrhage but the widespread use of invasive hepatobiliary procedures and improved recognition has increased its incidence. ${ }^{4}$ Hemobilia can be caused by traumatic and non-traumatic reason. The most common traumatic cause in Western countries is liver trauma, followed by iatrogenic injury during diagnostic and therapeutic hepatobiliary procedures (liver biopsy, percutaneous transhepatic cholangiography). ${ }^{5} \quad$ Non-traumatic causes include, inflammatory disorders( cholecystitis and pancreatitis) and gallstones. ${ }^{6,7}$ Tumors, vascular diseases such as aneurysm and bleeding disorders may also cause hemobilia. ${ }^{8}$

Pseudoaneurysms are differentiated from true aneurysms by the presence of a known cause such as trauma or inflammation, and by radiologic findings of aneurysmal dilatation in the setting of otherwise normal artery. Among 55 well-documented cases of hemobilia, $53 \%$ were originated in the liver, $23 \%$ in the gallbladder, $22 \%$ in the bile ducts and $2 \%$ in the pancreas . ${ }^{9}$ Pseudoaneurysm can occur in hepatic artery, less common in cystic artery. ${ }^{10}$ Postcholecystectomic hemobilia is one of the rarest vascular complication following laparoscopic cholecystectomy and usually occurs as a result of cystic or right hepatic arterial wall injury. ${ }^{6}$ Postcholecystectomic hemobilia usually occurs 4 weeks after operation. ${ }^{11}$ Common bile duct wall injury also cause hemobilia. ${ }^{6}$ Postcholecystectomic hemobilia due to vascular wall injury may be caused by physical, thermal and chemical injury during laparoscopy, but even it is not yet proved .Furthermore operation reports of the hemobilia patients frequently describe inflamed gallbladders and difficult cholecystectomies ${ }^{8}$ Clinical presentation of hemobilia can be 
variable and intermittent. ${ }^{3}$ Severe hemobilia resulting in hemodynamic instability or necessitating transfusion. ${ }^{12}$ Hemobilia is classically associated with Quincke's clinical triad (colicky abdominal pain,jaundice and gastrointestinal haemorrhage. ${ }^{13}$ However, most patients do not present with all four of these findings. ${ }^{4}$ Blood may clot in various locations depending on the amount and rate of bleeding, hematemesis or melena may occur. ${ }^{14}$ Common bile duct occlusion by blood clot may lead to obstructive jaundice. ${ }^{12}$ Obstruction of the pancreatic duct by the blood clots and reflux into the pancreatic duct leads to pancreatitis which is another causes of hemobilia ${ }^{15}$.Acute biliary symptoms and signs combined with acute upper gastrointestinal bleeding should strongly suggest the possibility of hemobilia. ${ }^{8}$ The clue to the diagnosis of Acute Pancreatitis due to hemobilia is a manifestation of UGI bleeding in the background of Acute Pancreatitis. ${ }^{16}$ The association of haemobilia and Acute Pancreatitis is rare. It has been reported after liver biopsy, percutaneous transhepatic billiary drainage \& hepatocellular carcinoma. ${ }^{17}$ For the diagnosis and evaluation of hemobilia sonography and computed tomography are helpful in assessing the possibility of tumor or stone disease. ${ }^{9}$ Another useful investigation is MRCP, which can show biliary dilatation and irregular filling defects, can distinguish blood from stones and sludge and may be helpful when the diagnosis is uncertain. ${ }^{8}$

The diagnosis of hemobilia is most frequently made by upper endoscopy or ERCP. ${ }^{19}$ If bile duct obstruction is suspected, early ERCP is advisable. ${ }^{14}$

ERCP and clot retrieval by balloon sweep was performed in addition to embolization. ${ }^{20}$ Angiography should be considered early in cases of trauma or known tumor for both diagnosis and therapy; it is useful in localizing the site of bleeding. ${ }^{8}$ Hemobilia from a hepatic artery source frequently requires transcatheter embolization via a right femoral artery approach or even hepatic artery ligation, which can result in liver failure in patients with underlying hepatic insufficiency.$^{12}$ Angiography may provide an opportunity for arterial embolization which can be simple \& safe . Vascular injuries during laparoscopic cholecystectomy may occur in $0.2 \%-0.5 \%$ cases and these injuries are also reported that they may cause hemobilia with pseudoaneurysmatic dilatation. ${ }^{8}$ In case of massive bleeding mortality rate can be as high as $50 \% .^{21}$

\section{Conclusion:}

Hemobilia after cholecystectomy and acute pancreatitis after hemobilia which resulted from liver trauma are previously and separately described conditions in the literature. But hemobilia rising from a pseudoaneurysm after cholecystectomy and causing acute pancreatitis is a rare condition. Our patient had a hemobilia which caused obstructive jaundice and acute pancreatitis one month after cholecystectomy \& our patient also had two vascular abnormalities.

\section{References:}

1. Baker KS, Tisnado J, Cho SR, Beachley MC. Splanchnic artery aneurysms and pseudoaneurysms: transcatheter embolization. Radiology. 1987;163(1):135-139
2. Huang CW, Hsu PI, Chan HH. Acute pancreatitis and cholangitis caused by hemobilia secondary to hepatocellualr carcinoma. J Intern Med Taiwan. 2013;24:500-504.

3. Sandblom P: Hemorrhage into the biliary tract following trauma "traumatic hemobilia". Surgery 1948, 24(3):571-586

4. Yoshida J, Donahue PE, Nyhus LM: Hemobilia: review of recent experience with a worldwide problem. Am J Gastroenterol 1987,82(5):448-453

5. Maeda A, Kunou T, Saeki S. Pseudoaneurysm of the cystic artery with hemobilia treated by arterial embolization and elective cholecystectomy. J Hepatobiliary Pancreat Surg. 2002;9(6):755-758

6. England RE, Marsh PJ, Ashleigh R, Martin DF. Case report: pseudoaneurysm of the cystic artery: a rare cause of haemobilia. Clin Radiol. 1998;53:72-75

7. Lee MJ, Saini S, Geller SC, Warshaw AL, Mueller PR. Pancreatitis with pseudoaneurysm formation: a pitfall for the interventional radiologist. Am J Roentgenol. 1991;156:97-98

8. Bloechle C, Izbicki JR, Rashed MY, El-Sefi T, Hosch SB Hemobilia: presentation, diagnosis, and management. Am J Gastroenterol 1994, 89:1537-1540

9. Bismuth H: Hemobilia. N Engl J Med 1973, 288:617-619.

10. Goodwin SC, Bansai V, Greaser LE, Stainken BF, McNamara TO. Prevention of Hemobilia during percutaneous biliary drainage: long Term follow up. Journal of Vascular and Interventional Radiology 1997,8(5):881-883

11. Alfonso Ribeiro, Hugh Williams, Gerald May, Fulmer Jack T, Spivey James R. Hemobilia Due to Hepatic Artery Pseudoaneurysm Thirteen Months After Laparoscopic Cholecystectomy Journal of Clinical Gastroenterology. 1998, 26(1):50-55

12. Monden M, Okamura J, Kobayashi N, Shibata N, Horikawa S, Fujimoto $\mathrm{T}$ et al: Hemobilia after percutaneous transhepatic biliary drainage. Arch Surg 1980, 115(2):161-164

13. Nakajima M,Hoshino H,Hayashi E. Pseudoaneurysm of the cystic artery associated with upper gastrointestinal bleeding.J Gastroenterol 1996;31(5):750-754

14. Kroser J, Rothstein RD, Kochman ML.Endoscopic management of obstructive jaundice caused by hemobilia. Gastrointest Endosc 1996, 44:618- 619

15. Paikos D, Katsinelos P, Kontodimou K. Acute recurrent pancreatitis complicating hemobilia in a patient with hepatocellular cancer and recipient of anticoagulants successful treatment with metal stent placement (pancreatitis complicating hemobilia). Pancreas. 2007;34(1):168-9.

16. Peña LR, Horn TL, Cross CB. Acute pancreatitis secondary to hemobilia after percutaneous liver biopsy. Therap Adv Gastroenterol. 2009;2 (3):165-8.

17. Van os EC, Petersen BT. Pancreatitis secondary to percutaneous liver biopsy-associated hemobilia. Am J Gastroenterol. 1996;91(3):577-80.

18. Cattan P, Cuillerier E, Cellier C, Cuenod CA, Roche A, Landi B et al.Hemobilia caused by a pseudoaneurysm of the hepatic artery diagnosed by EUS. Gastrointest Endosc 1999, 49(2):252-255

19. Cappell Ms, Marks M, Kirschenbaum H. Massive hemobilia and acalculous cholecystitis due to benign gallbladder polyp. Dig Dis Sci 1993, 38(6):1156-6

20. Alis H, Bozkurt MA, Oner OZ. Case report. Acute pancreatitis caused by postcholecystectomic hemobilia. BMC Gastroenterol. 2010;10:75

21. Sandblom P.Hemobilia Edited by: Charles C, Thomas. Springfield; 1972 